\title{
The Investigation of the Acute Effect of Craniocervical Flexion Exercise and Local Vibration on Semispinalis Cervicis and Multifidus Muscle Activation in a Healthy Individual
}

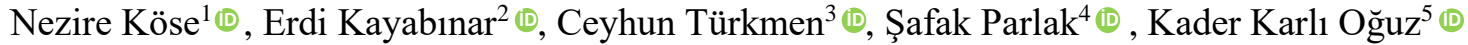

Submission Date: 9th of Aug., 2018 Acceptance Date: $11^{\text {th }}$ of March,2019 Pub.Date: 26t ${ }^{\text {h }}$ of April, 2919

\begin{abstract}
Aim: The aim of this study is to investigate the acute effects of craniocervical flexion exercise (CCFE) and vibration on the activation of the semispinalis cervicis and multifidus muscles in a healthy case. Materials and Methods: Our study was carried out on a healthy male without neck pain at the age of 21 at Hacettepe University Faculty of Health Sciences, Physiotherapy and Rehabilitation Department and Hacettepe University Faculty of Medicine, Radiology Department. First, the individual was taught craniocervical flexion exercise (CCFE). Later on, cervical MR images of the 3-T magnet MRG device were taken at the National Magnetic Resonance Research Center of Bilkent University. The sections obtained from the deep paravertebral region muscles by "T2 map color mapping method" and the muscle areas of activation were drawn by observation. T2 relaxation values associated with muscle activation were calculated. Later on, case was asked to perform CCF exercise with 10 seconds of contraction and 5 seconds of rest and 20 repetitions. MRI measurements were repeated after exercise. After a resting period of forty-five minutes, the images of the paravertebral muscles were recorded with MRI again before the vibration and the neck of the case was vibrated for 5 minutes. After the vibration, the MRI measurements were repeated and compared with the results of the first measurement. Results: It has been found that the results obtained immediately after the KSF exercise and after the application of vibration have some limited variations in the activation of the deep paravertebral neck muscles when compared to the results before CCF and vibration.

Conclusion: At the end of this study it was thought that the CCF exercise and the application of vibration may have a limited and positive effect on the deep paravertebral muscles and studies with different exercise protocol and vibration intensity on more cases are needed in order to clarify this effect more clearly.
\end{abstract}

Keywords: craniocervical flexion, vibration, fMRG, cervical paravertebral muscles.

\footnotetext{
${ }^{1}$ Nezire Köse (Corresponding Author). Hacettepe University, Faculty of Health Sciences, Department of Physical Therapy and Rehabilitation, 0312305 1576. e-mail: fztnezire@ yahoo.com.

${ }^{2}$ Erdi Kayabınar. Hacettepe University, Faculty of Health Sciences, Department of Physical Therapy and Rehabilitation.e-mail: erdikayabinar@gmail.com.

${ }^{3}$ Ceyhun Türkmen. Hacettepe University, Faculty of Health Sciences, Department of Physical Therapy and Rehabilitation.e-mail: fztceyhunturkmen@gmail.com.

${ }^{4}$ Şafak Parlak. Hacettepe University, Faculty of Medicine, Department of Radiology. e-mail: parlaksafak@gmail.com.

${ }^{5}$ Kader Karlı Oğuz. Hacettepe University, Faculty of Medicine, Department of Radiology. e-mail: karlioguz@yahoo.com.
} 


\section{Introduction}

Cervical region problems are the second most common issue after the lumbal region in general population and musculoskeletal system disorders, affecting close to $70 \%$ of the general population. These problems can be caused by reasons such as decreased muscle performance, weaker gravitational muscles with early fatigue, and neck pain developed as secondary to inability to stabilize the spinal cord completely (Jull, 2000; Boyd-Clark, Briggs \& Galea, 2002; Karaduman \& Tunca Y1lmaz, 2016).

Nowadays, evidence-based treatment methods for spinal pathologies and accompanying neck pain are directed towards the use of new exercises and different modalities. The most common exercise is stabilization exercises which create an activation on deep muscles of the neck region (Karaduman \& Tunca Y1lmaz, 2016; Kisner \& Colby, 2007; Şimşek, 2017; Brumitt, Matheson \& Meira, 2013). Stabilization exercises often aim to increase the activation of deep group muscles (especially longus colli, longus capitis, semispinalis cervicis and multifidus) and stabilization of the cervical spine (Şimşek, 2017). Another method used clinically to activate the cervical paravertebral muscles in recent years is the application of local vibration. Local vibration applications are a new method used in this area, which stimulates the muscle spindle to increase both proprioceptive input and muscle activity (Beinert, Keller, Taube \& 2015; Luo, McNamara \& Moran, 2005).

In the former studies; the activity of the superficial muscles (especially sternocloidomastoid and upper trapezoid) was found higher than deep muscles on patological and painful subjects, even though the activity of the deep group muscles in the healthy individuals was greater than the superficial muscles during normal joint movements in the cervical region (Cagnie, Dirks, Schouten, Parlevliet, Cambier \& Danneels, 2011; Falla, 2004; Falla, Jull \& Hodges, 2004). Superficial muscles tend to increase their activity as compensator in painful situations. The stabilizing ability of the normal healthy spinal cord decreases with painful conditions, and superficial muscles exhibit increased compensatory activity to reduce pain in spinal stance and movements that stimulate the pain (Cagnie, Dirks, Schouten, Parlevliet, Cambier \& Danneels, 2011; Falla, 2004). It is emphasized in literature that coactivations of deep flexor muscles (especially longus colli and longus capitis) and deep extensor muscles (especially semispinalis cervicis and multifidus) are important in maintaining neck stabilization (Jull, Sterling, Falla, Treleaven \& O'Leary, 2008). The aim in the treatment of neck problems should be to increase the strength and endurance of these muscles and to regain the function. In the clinic, as a stabilization exercise, mostly craniocervical flexion exercises 
are used, but when the literature is examined, there is no specific exercise approach developed for activating deep cervical extensor muscles specifically (Ünal, 2015). Only a few publications have reported that cranio-cervical extension can be performed for cervical extension (Jull, Sterling, Falla, Treleaven \& O’Leary, 2008; O’Leary, Cagnie, Reeve, Jull \& Elliott, 2011). It has been shown that craniocervical flexion exercises increase cervical stabilization and decrease spinal pathologies and pathologically induced pain while increasing the activity of the deep group muscles of the cervical spine (Cagnie, D’Hooge, Achten, Cambier \& Danneels, 2010; Falla, Jull, Dall'Alba, Rainoldi \& Merletti, 2003). In addition, a co-activation with stabilization exercises among the deep muscle groups providing stabilization in the lumbar region has been reported (Kisner \& Colby, 2007; Şimşek, 2017; Jull, Sterling, Falla, Treleaven \& O’Leary, 2008), there is no published study investigating the co-activation of craniocervical flexion exercises among all deep group muscles stabilizing the cervical region.

In the literature review of local vibration which is another application that is increasingly used to activate cervical paravertebral muscles in recent years, there are publications showing that application of vibration increases sensorimotor function in patients with neck pain and that the vibration applied to other muscles of the body also has a strengthening effect on the muscles (Beinert, Keller \& Taube, 2015; Luo, McNamara \& Moran, 2005). In these publications; it has been shown that vibration can acutely increase isometric and dynamic maximal contraction force in the vastus lateralis, wrist extensor and ankle dorsi flexor muscles (Luo, McNamara \& Moran, 2005; Curry \& Clelland, 1981; Humphries, Warman \& Purton, 2004). In these studies, it is seen that local vibrations are applied at frequencies between 26 and $100 \mathrm{~Hz}$ and amplitudes between 0.3 and $1.8 \mathrm{~mm}$ for this purpose (Curry \& Clelland, 1981; Humphries, Warman \& Purton, 2004). Although there have been some studies to prove improving muscle strength by applying local vibration, there have been no studies investigating the effect of vibration on paravertebral muscles on muscle strength.

In addition, it is also clear that there is a need for advanced imaging methods to evaluate the effects of stabilization exercises and vibration on the muscles in the neck region. However, work done in this area is limited. The necessity of invasive methods for achieving muscle selectivity rather than superficial EMG studies makes it difficult to use EMG as an evaluation method. Therefore, the EMG method can not be accepted as a practical method to be used in the clinic (Cagnie, Dirks, Schouten, Parlevliet, Cambier \& Danneels, 2011; Cagnie, D’Hooge, Achten, Cambier \& Danneels, 2010). In a study of Falla in 2004, the nasopharyngeal EMG method was used to assess the longus colli and longus capitis which are the deep cervical 
muscles. Because this method is invasive and can not be used to measure paravertebral muscles and other disadvantages of EMG methods, the investigators directed to use magnetic resonance imaging (MRI) method that is non-invasive and can provide muscle selectivity (Falla, Jull \& Hodges, 2004).

Increasing MRI studies in recent years have evaluated the architectural and physiological changes of muscles during activity, thereby providing an objective indication of muscle activation during and after the activity (Cagnie, Elliott, O'Leary, D'Hooge, Dickx \& Danneels, 2011; Cagnie, Dickx, Peeters, Tuytens, Achten, Cambler \& Danneels, 2008). In the literature, there are studies evaluating the effects of craniocervical flexion exercises on longus colli and longus capitis muscles (Cagnie, Dirks, Schouten, Parlevliet, Cambier, Danneels, 2011; Cagnie, D’Hooge, Achten, Cambier, Danneels \& 2010; Cagnie, Elliott, O’Leary, D'Hooge, Dickx \& Danneels, 2011) and craniocervical extensor exercises on paravertebral muscles (O’Leary, Cagnie, Reeve, Jull \& Elliott, 2011). However, no study evaluating the effects of craniocervical flexion exercises and local vibrations on the cervical paravertebral muscles with MRI was found. For this reason, this case report was planned as a pilot study to evaluate the acute effects of these exercises on a healthy subject.

\section{Materials and Methods}

A 21-year-old male with a weight of $90 \mathrm{~kg}$ and a height of $182 \mathrm{~cm}$ was evaluated in our study. Inclusion criteria in case selection are; do not have any surgical application of vertebral colon, inflammatory disease, rheumatologic disease, history of malignancy, congenital spinal cord anomaly, congenital and/or gained spinal deformities, radiculopathy, myelopathy and/or other neurological disorders, vestibular disorder or trauma story, and for MRI measurements do not have pacemakers, metal implants, non-removable piercings, cochlear or ear implants and aneurysm clipping surgery story and not being claustrophobic.

In our study, all evaluations were performed with a 3-T magnet (Siemens Magnetom Trio System) MR Imager. When images were obtained, 12-channel head and 8-channel neck coils were used together. The case relaxed and laid on the back. The head was positioned in the neutral position without rotation, lateral flexion or excessive lordosis. Images were obtained with T2-weighted, T2A sagittal, T2A axial and axial T2 mapping, with the same values on the same plane each time. T2 color maps were obtained by postprocessing. In the T2 color map with ROI were plotted freely taking into account the muscle areas on axial slices, and the mean T2 relaxation values were calculated (Figure 1). 
T2 relaxation time is a measurement technique that has become increasingly widespread in recent years. When the tissue is placed in a strong magnetic field, the magnetic reactivity of hydrogen atoms in water and fat tissue is increasing. Within the magnetic field, each of the hydrogen atoms begins oscillating in a synchronous manner with a particular radiofrequency. These oscillations generate a magnetic signal and can be measured electronically. After a certain period of time, hydrogen atoms that oscillate together show different oscillations and magnetic signals begin to fall off. The decline in nuclear magnetic resonance signals due to the reduction of synchronous oscillations is called transverse or T2 relaxation. The rise and fall caused by the magnetic behavior of these hydrogen atoms is measured in milliseconds. The muscle activation following exercise increases the intracellular and extracellular water contents of the myofibrils. The increase in phosphate, lactate and sodium concentrations accumulated by muscle activation in the cytoplasm leads to an increase in the amount of water in the myofibrils due to osmolarity. The increased amount of water also causes an increase in the T2 relaxation time measured on the muscle area. In the literature, there are publications showing that the increase in $\mathrm{T} 2$ relaxation time and the increase in muscle activity are strong correlates (O’Leary, Cagnie, Reeve, Jull \& Elliott, 2011; Cagnie, D’Hooge, Achten, Cambier \& Danneels, 2010; Cagnie, Elliott, O’Leary, D’Hooge, Dickx \& Danneels, 2011; Fleckenstein, Canby, Parke \& Peshock, 1988; Meyer \& Prior, 2000).

In our study; MRI measurements were recorded immediately before and immediately after the application to determine changes in these activations that occurred in the muscles after exercise and vibration applications. The case was trained on craniocervical flexion exercise for 5 times, during 10 days before the test day and perform the exercise at $30 \mathrm{mmHg}$.

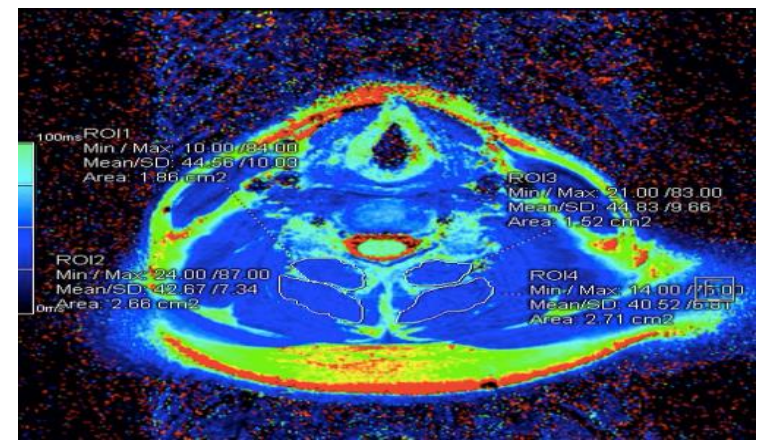

Figure 1.a. MRI results before exercise. 


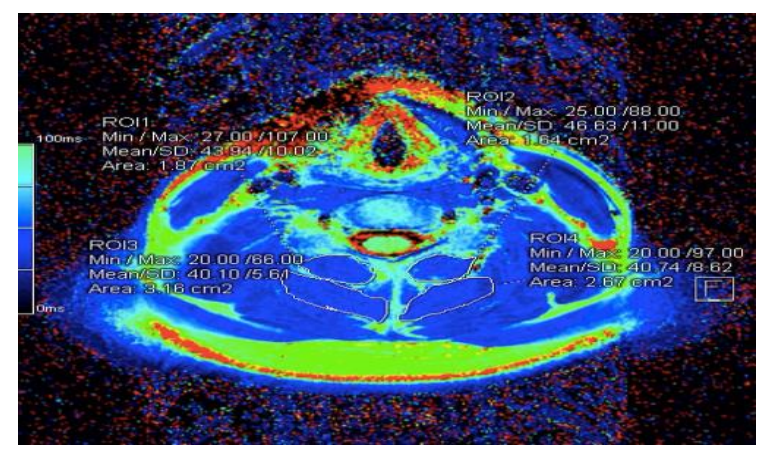

Figure 1.b. MRI results after exercise.

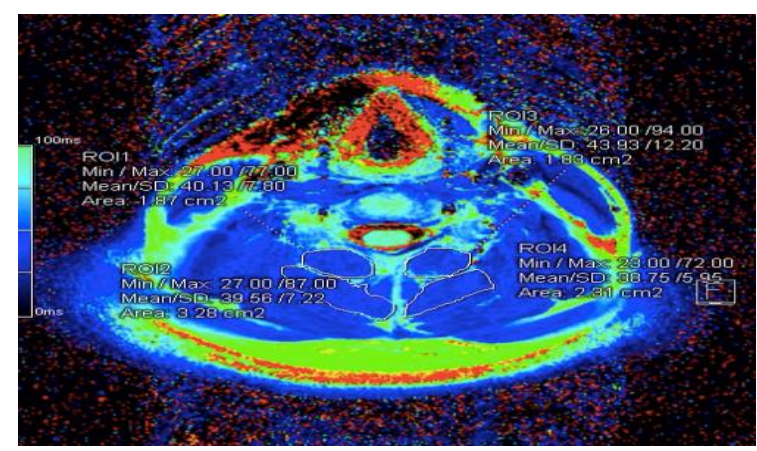

Figure 1.c. MRI results before vibration.

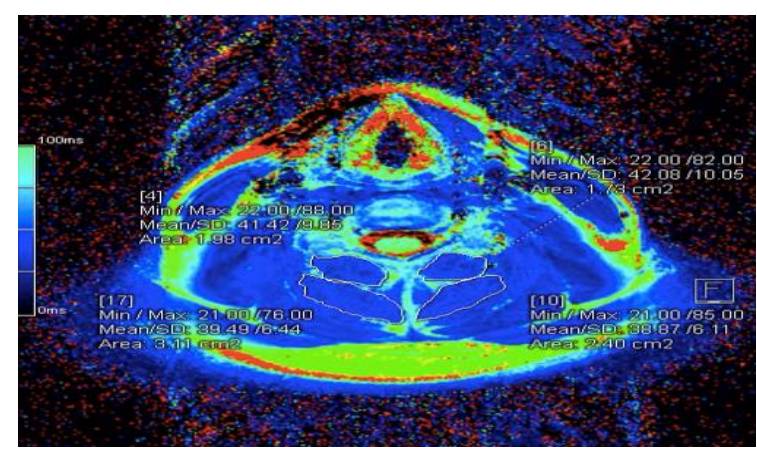

Figure 1.d. MRI results after vibration.

On the test day, after the first MRI evaluation, case was asked to complete the craniocervical flexion exercises at $30 \mathrm{mmHg}$ on the stabilizer device with 10 -s. contraction and 5-s resting times and 20 repetitions (Figure 2). 


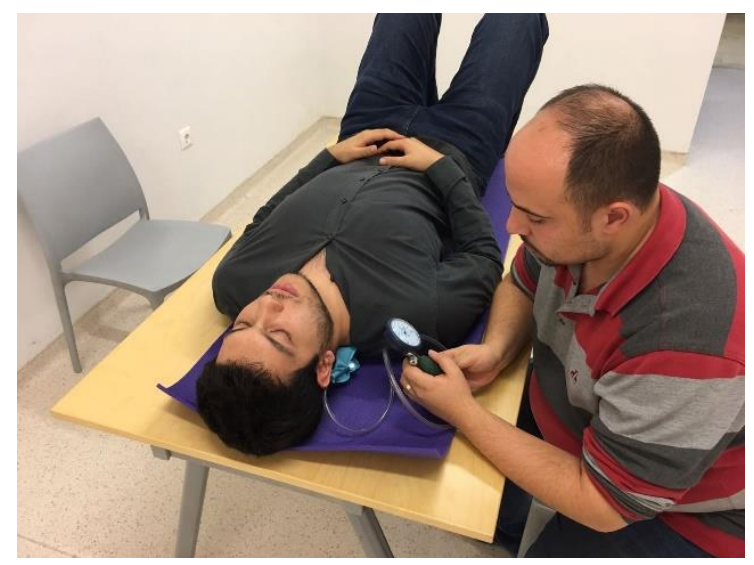

Figure 2. Craniocervial flexion exercise.

Immediately after the exercises MRI was evaluated again. Since it was stated that the muscle activation level could be returned to the baseline level after at least 45 minutes (Cagnie, Dirks, Schouten, Parlevliet, Cambier \& Danneels, 2011; Cagnie, D’Hooge, Achten, Cambier \& Danneels, 2010; Cagnie, Elliott, O’Leary, D’Hooge, Dickx \& Danneels, 2011; Cagnie, Dickx, Peeters, Tuytens, Achten, Cambler \& Danneels, 2008), waited for 45 minutes between the end of the exercise and the pre-vibration evaluation. After 45 minutes, the case underwent MRI evaluation before vibration. Subsequently, vibration of the paravertebral muscles of the neck was applied at frequencies of $60-80 \mathrm{~Hz}$ and at a frequency of $1.00 \mathrm{~mm}$ to prevent adaptation with the vibrator for 5 minutes (Figure 3). Immediately after the application of the vibration, final MRI evaluation was performed.

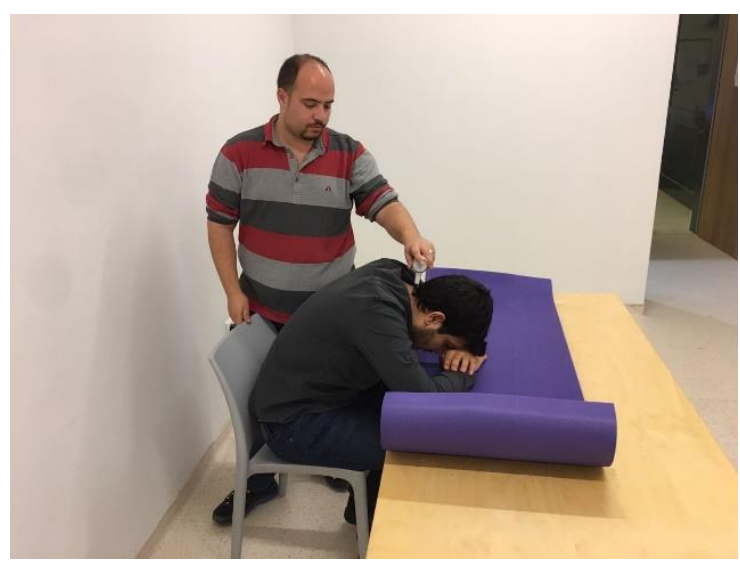

Figure 3. Application of vibration. 


\section{Results}

At the end of the study, $\mathrm{T} 2$ relaxation times of the semispinalis cervicis and multifidus muscles were calculated in milliseconds and the results are shown in table 1. According to the results obtained immediately after exercise and vibration applications, a slight change was observed in semispinalis cervicis and multifidus muscles. A decrease in the left side was observed when the muscle activations increased on the right side. After vibration, muscle activation in the semispinalis cervicis, increase in the right side, decrease in the left side was observed and in the multifidus muscle; on the contrary, decrease in right and increase in left was found. These changes were minimal, and in the semispinalis cervicis, we can say no change was observed.

Table 1. T2 relaxation times

\begin{tabular}{llllllll}
\hline & \multicolumn{2}{l}{ Pre-exercise } & \multicolumn{2}{l}{ After exercise } & \multicolumn{2}{l}{ Pre-vibration } & \multicolumn{2}{c}{ After vibration } \\
\hline Right & Left & Right & Left & Right & Left & Right & Left
\end{tabular}

\begin{tabular}{|c|c|c|c|c|c|c|c|c|c|}
\hline 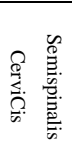 & $\mathrm{T} 2(\mathrm{~ms})$ & 40.5 & 42.7 & $\begin{array}{c}40.7 \\
\uparrow\end{array}$ & 40.1 & 38.8 & 39.6 & $\begin{array}{c}38.9 \\
\uparrow\end{array}$ & 39.5 \\
\hline 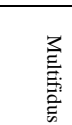 & $\mathrm{T} 2(\mathrm{~ms})$ & 44.8 & 44.6 & $\begin{array}{l}46.6 \\
\uparrow\end{array}$ & $\begin{array}{r}43.9 \\
\downarrow\end{array}$ & 43.9 & 40.1 & $\begin{array}{c}42.1 \\
\downarrow\end{array}$ & 41.4 \\
\hline
\end{tabular}

$\uparrow:$ Increase after the application. $\quad \downarrow$ : Decrease after application

\section{Conclusion}

At the end of this case study, it was observed that cranioservical flexion exercise and vibration to the cervical paravertebral region caused minimal changes in $\mathrm{T} 2$ relaxation times of semispinalis cervicis and multifidus muscles acutely. It has been noted in the literature that the use of craniocervical flexion exercise in the treatment of neck pathologies and neck pain is increasingly used to improve neck stability and spinal stability (Kisner \& Colby, 2007; Şimşek, 2017; Brumitt, Matheson \& Meira, 2013). However, the effects of craniocervical flexion exercise have been shown in the deep group flexor muscles such as longus colli and longus capitis (Falla, Jull, Dall'Alba, Rainoldi \& Merletti, 2003; Cagnie, Dickx, Peeters, Tuytens, Achten, Cambler \& Danneels, 2008). Studies investigating the changes in the activity levels of the neck muscles in the neck pathologies and accompanying pain found that the decreased 
activity in both the flexor and extensor muscles are associated with pain (Cagnie, Dirks, Schouten, Parlevliet, Cambier \& Danneels, 2011; Falla, 2004; O’Leary, Cagnie, Reeve, Jull \& Elliott, 2011; Kristjansson, 2004). For this reason, it is necessary to plan exercises to control pain in order to control the activity of both deep muscle groups. Cagnie et al. in a study conducted in 2011, the activity levels measured by the T2 relaxation times of the longus colli and longus capitis muscles showed a larger increase than the sternocloidomastoid muscle after the cranioservical flexion exercises, however in painful cases, there was a decrease in longus colli and longus capitis activity and an increase in sternocloidomastoid muscle activity compared to healthy control (Cagnie, Dirks, Schouten, Parlevliet, Cambier \& Danneels, 2011). In a study conducted by O'Leary et al. in 2011 showed that $\mathrm{T} 2$ relaxation times were increased in multifidus and semispinalis cervicis muscles after craniocervical extension exercise in healthy and mechanically painful neck patients (O’Leary, Cagnie, Reeve, Jull \& Elliott, 2011). In the study performed by Falla, effects of cranioservical flexion exercises on the deep group flexor muscles was measured with nasal EMG, the activity of the deep group flexor muscles was found to increased from $22 \mathrm{mmHg}$ to $30 \mathrm{mmHg}$ (Falla, Jull \& Hodges, 2004). In a study conducted in 2010 , they examined the effects of different cervical flexion exercises on longus colli, longus capitis and sternocloidomastoid muscles and found no increase in deep group muscle activation in cervical flexion exercise, but they found an increase in activation of longus colli and longus capitis muscles by craniocervical flexion exercise and this increase was found to be significant in longus capitis compared to sternocloidomastoid muscle (Cagnie, D'Hooge, Achten, Cambier \& Danneels, 2010). It has been determined that the statistical significance of the change in T2 relaxation times after exercise is greater than $3.5 \mathrm{~ms}$ (Cagnie, Dickx, Peeters, Tuytens, Achten, Cambler \& Danneels, 2008). In studies where the changes in T2 relaxation times were significant, it was found that this difference reached values greater than $3.5 \mathrm{~ms}$ (O’Leary, Cagnie, Reeve, Jull \& Elliott, 2011; Cagnie, Dickx, Peeters, Tuytens, Achten, Cambler \& Danneels, 2008). Our study showed a decrease on the left while the relaxation times of T2 were increased on the right side of semispinalis cervicis and multifidus right after exercise. However, it was determined that none of these differences could be attributed to the $3.5 \mathrm{~ms}$, which is statistically significant, as seen in other studies. Findings in this study suggest that co-contractions may have occurred during craniocervical flexion exercises. However, it was decided that further studies should be done because these results were obtained from only one case and the increases were minimal. It was also thought that the frequency and the dose of the CCF exercise might be too high, because it was noticed that the individual showed signs of 
fatigue towards the end of the exercise. It was thought that the fall in muscle activity on the left side after exercise could be related to this fatigue, and the reason for not being on the right side may be related to the right side is the dominant side of the case.

In addition to this information, stabilization exercises for the neck in the clinic usually start with craniocervical flexion exercises and craniocervical extensor exercises are either included or not included in treatment programs at very advanced stages (Jull, Sterling, Falla, Treleaven \& O’Leary, 2008). However, the literature suggests that activation of deep group flexor muscles by craniocervical flexion exercises and the activation of deep group extensor muscles by craniocervical extensor exercises could be obtained, the activities of these muscles are diminishing together in cervical pathologies and accompanying pain and co-activation of both muscle groups is required to achieve spinal stabilization and so it is thought that the exercises that these two groups of muscles work together should be planned. In this way, the activity of the anterior and posterior group muscles of the neck can be increased in the same way to provide a complete co-activation for neck stabilization. For this reason, there is a need for further studies to examine the activities of exercise programs created by combining craniocervical flexion and extension exercises in both groups of deep muscles.

In our study, similar results were obtained as an acute effect at the end of vibration application, which is another application that we thought would increase the activation of deep cervical stabilizer muscles. Immediately after the application of the vibration, the semispinalis cervicis muscle on the right and the multifidus muscle on the left side showed a slight increase in the activity levels measured by the $\mathrm{T} 2$ relaxation period. It was also found that these changes in the semispinalis cervicis were negligible. There was a decrease on the right side of the multifidus. When the causes of these results were considered, it was concluded that the effects of local vibration may not have been effective due to the deep placement of both muscles, the amount of frequency may not have been sufficient, the acute effects of a single session application may have been inadequate. Chronic effects that may occur with long-term applications need to be examined.

In a study conducted by Curry, it was determined that high frequency vibration application could acutely increase isometric strength of wrist extensors (Curry \& Clelland, 1981). Luo et al., in their review, investigated the study of vibration to increase acute dynamic contraction and maximal isometric contraction in wrist extensors and foot dorsi flexors and they found long-term application of local vibration in order to increase the tonic vibration reflex, muscle spindle excitability and sensorimotor functions, thereby creating a strengthening effect 
on the muscles (Luo, McNamara \& Moran, 2005). This result is parallel to our conclusions and interpretations.

As a result of this case study, we find that there may be minimal increases in activation of semispinalis cervicis and multifidus muscles after craniocervical flexion exercise and local vibration and there is a need for further investigations in this area. 


\section{References}

Beinert K., Keller M., Taube W. (2015). Neck muscle vibration can improve sensorimotor function in patients with neck pain. The Spine Journal, 15(3), 514-521.

Boyd-Clark LC., Briggs CA., Galea MP. (2002). Muscle spindle distribution, morphology and density in longus colli and multifidus muscles of the cervical spine. Spine, 27, 694-701.

Brumitt, J., Matheson, J.W., Meira, E.P. (2013). Core Stabilization Exercise Prescription, Part I: Current Concepts in Assessment and Intervention. Sports Physical Therapy, 5(6), 504-509.

Cagnie, B., Dickx, N., Peeters, I., Tuytens, J., Achten, E., Cambler, D., et al. (2008). The use of functional MRI to evaluate cervical flexor activity during different cervical flexion exercises. $J$. Appl Physiol, 104, 230-235.

Cagnie, B., D'Hooge, R., Achten, E., Cambier, D., Danneels, L. (2010). A magnetic resonance imaging investigation into the function of the deep cervical flexors during the performance of craniocervical flexion. J Manipulative Physiol Ther, 33, 286-291.

Cagnie, B., Dirks, R., Schouten, M., Parlevliet, T., Cambier, D., Danneels, L. (2011). Functional reorganization of cervical flexor activity because of induced muscle pain evaluated by muscle functional magnetic resonance imaging. Manual Therapy, 16, 470-75.

Cagnie, B., Elliott, J., O'Leary, S., D'Hooge, R., Dickx, N., Danneels, L. (2011). Muscle Functional MRI as an imaging tool to evaluate muscle activity. Journal of orthopaedic \& sports physical therapy, 41(11), 896-903.

Curry EL., Clelland JA. (1981). Effects of the asymmetric tonic neck reflex and high-frequency muscle vibration on isometric wrist extension strength in normal adults. Phys Ther, 6(4), 48795.

Falla D., Jull G., Dall'Alba P., Rainoldi A., Merletti R. (2003). An electromyographic analysis of the deep cervical flexor muscles in performance of craniocervical flexion. Phys Ther, 83, 899-906.

Falla, D. (2004). Unravelling the complexity of muscle impairment in chronic neck pain. Manual Therapy, 9(3), 125-133.

Falla DL., Jull GA., Hodges PW. (2004). Patients with neck pain demonstrate reduced electromyographic activity of the deep cervical flexor muscles during performance of the craniocervical flexion test. Spine, 29, 2108-2114.

Fleckenstein, J.L., Canby, R.C., Parke, R.W., Peshock, R.M. (1988). Acute effects of exercise on MR imaging of skeletal muscle in normal volunteers. AJR Am. J., 151, 231-237.

Humphries B., Warman G., Purton J. (2004). The influence of vibration on muscle activation and rate of force development during maximal isometric contractions. J Sports Sci Med, 3, 16-22.

Jull GA. (2000). Deep cervical muscle dysfunction in whiplash. World Congres On WhiplashAssociated Disorders İn Vancouver, British Columbia, Canada. J Musculoskelet Pain, 8, 14354.

Jull G., Sterling M., Falla D., Treleaven J., O'Leary S. (2008). Therapeutic Exercise for Cervical Disorders: Practise Pointers: Whiplash, Headache, and Neck Pain (pp:207-229). Edinburgh: Churchill Livingstone.

Ün Yıldırım, N., Özengin, N., (2016). Fizyoterapi ve Rehabilitasyon 1. A.A. Karaduman \& Ö.Tunca Y1lmaz (Eds), Kor stabilite (pp:219-227). Ankara: Hipokrat Kitabevi \& Pelikan Kitabevi.

Kisner C., Colby L.A. (Eds.) (2007). Therapeutic Exercise: Foundations and Techniques. (Sixth Edition). Philadelphia: F.A. Davis Company.

Kristjansson E. (2004). Reliability of ultrasonography for the cervical multifidus muscle in asymptomatic and symptomatic subjects. Man Ther, 9, 83-88.

Luo, J., McNamara, B., Moran K. (2005). The use of vibration training to enhance muscle strenght and power. Sports Med, 35(1), 23-41.

Meyer RA., Prior BM. (2000). Functional magnetic resonance imaging of muscle. Exerc Sport Sci Rev, 28, 89-92.

O'Leary, S., Cagnie, B., Reeve, A., Jull, G., Elliott, JM. (2011). Is there altered activity of the extensor muscle in chronic mechanical neck pain? A functional magnetic resonance imaging study. Arch Phys Med Rehabil, 92, 922-934.

Şimşek, İ.E. (Ed.) (2017). Omurga. (1.Bask1). Ankara, Hipokrat Kitabevi.

Ünal E. (Ed.) (2015). Fizyoterapide Kanıta Dayalı Egzersiz Yaklaşımları. Ankara, Pelikan Kitabevi. 